\title{
Neighborhoods, Social Support, and African American Adolescents' Mental Health Outcomes: A Multilevel Path Analysis
}

\author{
Noelle M. Hurd \\ University of Virginia
}

\author{
Sarah A. Stoddard and \\ Marc A. Zimmerman \\ University of Michigan
}

\begin{abstract}
This study explored how neighborhood characteristics may relate to African American adolescents' internalizing symptoms via adolescents' social support and perceptions of neighborhood cohesion. Participants included 571 urban, African American adolescents (52\% female; $M$ age $=17.8$ ). A multilevel path analysis testing both direct and indirect effects of neighborhood characteristics on adolescents' mental health outcomes was conducted. Higher neighborhood poverty and unemployment rates predicted greater internalizing symptoms via lower cumulative social support and perceptions of neighborhood cohesion. In contrast, higher concentrations of African American and residentially stable residents in one's neighborhood related to fewer internalizing symptoms among adolescent residents via greater cumulative social support and perceptions of neighborhood cohesion. Implications of these findings are discussed.
\end{abstract}

Despite widespread agreement among social scientists regarding the role of context in the development of adolescents' mental health problems (Zahn-Waxler, Klimes-Dougan, \& Slattery, 2000), few researchers have considered contextual predictors of adolescent psychopathology beyond family or peer influences. Of course, substantial findings have documented the potential of family and peer processes to shape adolescents' mental health outcomes, yet we know less about how community or neighborhood environments may affect social processes related to youths' psychological well-being (Leventhal \& Brooks-Gunn, 2000). Furthermore, researchers who have undertaken explorations of neighborhood effects on youths' psychosocial outcomes have primarily considered direct effects of neighborhood characteristics while controlling for aspects of youths' social relationships as opposed to considering how these social resources may transmit the effects of neighborhood characteristics on individual outcomes (for exceptions, see Deng et al., 2006; Kohen, Leventhal, Dahinten, \& McIntosh, 2008; Wickrama \& Bryant, 2003; Xue, Leventhal, BrooksGunn, \& Earls, 2005).

This research was supported by the National Institute on Drug Abuse, Grant DA07484 to the third author. We thank the youth for participating in this study and the Flint Community Schools for their support. We also thank the four anonymous reviewers for their helpful comments on previous versions of this manuscript.

Correspondence concerning this article should be addressed to Noelle M. Hurd, Department of Psychology, University of Virginia, 102 Gilmer Hall, P.O. Box 400400, Charlottesville, VA 22904-4400. Electronic mail may be sent to nh3v@virginia.edu.
Guided by Bronfenbrenner's (1979) ecological model, researchers are increasingly reporting the importance of considering how neighborhood environments may operate via more proximate mechanisms to influence individual mental health outcomes. In addition to considering how neighborhoods may shape interpersonal forces in youths' lives, some researchers have also documented the significance of assessing how neighborhood characteristics relate to adolescents' outcomes by shaping adolescents' perceptions of their neighborhoods. Researchers have found that adolescents' subjective perceptions of their neighborhoods may mediate the effects of neighborhood demographics on adolescents' internalizing and externalizing behavior (Aneshensel \& Sucoff, 1996; Latkin \& Curry, 2003; Ross \& Mirowsky, 2009). Therefore, considering how adolescents experience their neighborhood appears to be a fruitful line of investigation in efforts to better understand how neighborhood characteristics may affect adolescent development. In this study, we tested a multilevel path model to examine how neighborhood characteristics may affect urban, African American adolescents' mental health outcomes via their influence on adolescents' cumulative social support and perceptions of neighborhood cohesion.

(C) 2012 The Authors

Child Development (c) 2012 Society for Research in Child Development, Inc. All rights reserved. 0009-3920/2013/8403-0009

DOI: $10.1111 /$ cdev. 12018 
Internalizing Symptoms Among African American Late Adolescents

Internalizing disorders (i.e., anxiety and depression) are common among adolescents, particularly older adolescents (Zahn-Waxler et al., 2000). In fact, researchers have found lifetime prevalence rates of some internalizing disorders among late adolescents comparable to lifetime prevalence rates among adults (Lewinsohn, Hops, Roberts, Seeley, \& Andrews, 1993). Additionally, researchers have found that symptoms of anxiety and depression (even subclinical presentations) in adolescence predict later depressive disorders (Breslau, Schultz, \& Peterson, 1995; Gotlib, Lewinsohn, \& Seeley, 1995). Moreover, researchers have found a continuity of depressive disorders from late adolescence into adulthood (Rao, Hammen, \& Daley, 1999). Comparative studies between African Americans and Whites suggest that although African Americans may demonstrate somewhat lower prevalence rates of depression and anxiety disorders over the life course, African Americans suffering from depression or anxiety disorders tend to experience greater mental illness severity and chronicity, and report greater functional impairment in comparison to their White counterparts (Himle, Baser, Taylor, Campbell, \& Jackson, 2009; Williams et al., 2007).

Specific to African American late adolescents and emerging adults, researchers have found elevated depressed mood among African Americans in comparison to Whites and Asians (Gore \& Aseltine, 2003). Research findings indicate that these differences may be attributable to social inequalities, particularly in light of African American youths' overrepresentation in economically disadvantaged communities and greater exposure to concentrated poverty in comparison to Whites (Murry, Berkel, Gaylord-Harden, Copeland-Linder, \& Nation, 2011). In support of this notion, research with a nationally representative sample of Black and White adolescents found that Black adolescents who were exposed to family and community poverty demonstrated the most psychological distress of all participants (Wickrama, Noh, \& Bryant, 2005). Researchers argue that the additional stressors faced by urban, economically disadvantaged, African American late adolescents may exacerbate stressful experiences associated with developmental transitions and result in elevated internalizing symptoms among African American youths during this developmental period (Zimmerman, Ramírez-Valles, \& Maton, 1999). The importance of investigating potential neighborhood effects on African American late adolescents' mental health is underscored by research suggesting that neighborhood characteristics may be more likely to influence youths' outcomes during this developmental period as a result of late adolescents' increased independence and greater opportunities for unsupervised exploration of their neighborhoods (Brooks-Gunn, Duncan, Leventhal, \& Aber, 1997).

\section{Neighborhoods, Social Support, and Mental Health}

Researchers have posited various theories explaining how neighborhood factors may influence the psychological well-being of residents. Wilson (1987, 2009) argues that high rates of unemployment and concentrated poverty in inner-city neighborhoods lead neighborhood residents to feel less selfefficacious and more hopeless and helpless. Consistent with family stress models, research findings suggest that neighborhood-level economic stressors may create conditions that stress residents and contribute to mental health problems among residents (Katz, Esparza, Carter, Grant, \& Meyerson, 2012; Latkin \& Curry, 2003; Ross \& Mirowsky, 2009; Sampson \& Morenoff, 1997). In addition to direct effects of exposure to neighborhood disadvantage on adolescents' psychological distress (Wickrama \& Bryant, 2003), community stressors associated with concentrated poverty may negatively affect adults' personal resources and interfere with their ability to support the youth in their families and communities (Deng et al., 2006; Pinderhughes, Nix, Foster, \& Jones, 2001; Wickrama \& Bryant, 2003). Building on this hypothesis, Wilson and others (Bell \& Jenkins, 1993; Sampson \& Groves, 1989) have suggested that neighborhood socioeconomic disadvantage breeds social disorder which may contribute to symptoms of anxiety and depression among child and adolescent residents.

Although primarily advanced to connect neighborhood characteristics to deviance and violence (Sampson \& Groves, 1989), social disorganization theory also may be relevant for explaining neighborhood effects on other psychosocial outcomes, such as psychological distress. According to social disorganization theory, neighborhood socioeconomic disadvantage lowers neighborhood cohesion and social control, and as a result, impinges upon the ability of neighborhood residents to promote positive youth outcomes. Massey and Denton (1993) argue that in socially disorganized neighborhoods, residents are more likely to mistrust their neighbors, stay indoors, limit contact with friends and family, and avoid public participation in the community. Consistent with theses hypotheses, researchers have found associations between neighborhood disadvantage and 
decreased social ties with neighbors (Geis \& Ross, 1998; Small, 2007). Although one consequence of a less supportive and nurturing community may be increased youth violence and delinquency, another equally plausible (and possibly co-occurring) outcome is increased psychological distress among residents (Wickrama \& Bryant, 2003). Accordingly, concentrated neighborhood economic disadvantage may weaken intergenerational social ties, resulting in higher levels of internalizing symptoms among youth residents.

Notably, other factors such as higher levels of residential stability may have the opposite effect by creating more cohesive and socially organized communities. Consistent with this notion, researchers have found that neighbors who are less mobile may be more likely to invest in social relationships in their local communities (Sampson \& Groves, 1989; Sampson \& Morenoff, 1997; Sampson, Raudenbush, \& Earls, 1997). These neighborhood relationships are what Coleman (1988) refers to as social capital. According to Furstenberg (1993), the neighborhood setting is a potential reservoir of social capital. Coleman (1988) discusses the ways in which social capital can contribute to more positive resident outcomes via norm setting, reciprocal obligations, and opportunities for sharing information. An additional form of social capital is social support. Social support is a form of social capital that individuals can draw upon to help them cope with daily stressors (Dominguez \& Watkins, 2003).

Researchers have consistently documented positive associations between social support and psychological well-being among adult and youth samples (Hussong, 2000; Newman, Newman, Griffen, O'Connor, \& Spas, 2007), as well as inverse associations between social support and depression (Newman et al., 2007; Pierce, Frone, Russell, Cooper, \& Mudar, 2000). In particular, research findings suggest that social support may help protect youth against the negative effects of stressors and promote more positive mental health outcomes (Hussong, 2000; Newman et al., 2007). Overall, much of the work focusing on the promotive effects of social support on psychological health has emphasized the role of perceived support with a specific emphasis on emotional and instrumental support and individuals' beliefs that they are members of communicative and caring networks (Turner \& Brown, 2010). In this study, we were particularly interested in perceived support from multiple sources (i.e., parents, peers, and nonparental adults) and adolescents' perceptions of the supportiveness and cohesiveness of their neighborhoods as we expected that these indicators of support would be most likely to influence adolescents' psychological health.

Previous research suggests that social support from a variety of different sources may yield comparable benefits on adolescents' psychological health (Colarossi \& Eccles, 2003; Munsch \& Blyth, 1993), suggesting that multiple important relationships in adolescents' lives have the potential to reduce adolescents' risk of mental illness through the provision of support. Further, researchers have suggested that the benefits of support from multiple important individuals in adolescents' lives are additive as opposed to compensatory (i.e., the absence of support from one source is not compensated for by support from another source; Garnefski \& Diekstra, 1996). Therefore, adolescents may reap the greatest psychological benefits when they are receiving substantial support from a number of important individuals in their lives (e.g., parents, peers, and nonparental adults). Correspondingly, in this study, we focused on cumulative social support from multiple important sources. Further, we assessed the extent to which adolescents perceived their neighborhoods to be supportive and cohesive. Similar to research findings related to support from specific individuals, researchers have documented inverse associations between adolescents' perceptions of their neighborhoods as supportive and their internalizing symptoms (Aneshensel \& Sucoff, 1996). Yet researchers have differed in their approaches to measuring neighborhoods.

\section{Measuring Neighborhoods}

The measurement of neighborhood characteristics has primarily relied on census data that have been aggregated to either the block group or tract level (Leventhal \& Brooks-Gunn, 2000). Given the tendency of neighborhood factors to be highly correlated, some researchers have opted to create composite measures of neighborhood disadvantage to avoid issues of multicollinearity (Cook, Shagle, \& Degirmencioglu, 1997; Sampson et al., 1997). Yet composite measures of neighborhood disadvantage may be problematic because they reduce the available information (Upchurch, Aneshensel, Sucoff, \& Levy-Storms, 1999). These composite variables do not allow for a more nuanced understanding of the ways in which specific neighborhood factors may affect the well-being of neighborhood residents.

A primary example of a neighborhood characteristic that may deserve more in-depth examination is the racial and ethnic composition of a neighborhood. Wilson (2009) underscores how racism, 
housing discrimination, redlining (mortgage restrictions based on the racial composition of a neighborhood), labor market shifts, employment discrimination, and a variety of other institutional practices and policies have created highly segregated, impoverished, urban communities. This segregation of urban poverty is reflected in statistics showing that in comparison to their White peers, African American children are about 10 times more likely to live in neighborhoods where $30 \%$ or more of the residents are poor (Duncan, Brooks-Gunn, \& Klebanov, 1994). In fact, despite declines in overall rates of concentrated poverty in the 1990s, African Americans remain the largest racial or ethnic group living in high-poverty neighborhoods (Jargowsky, 2005).

In light of the literature on segregated urban poverty (Massey \& Denton, 1993), a number of researchers have used the percentage of African American residents in a neighborhood as an indicator of neighborhood disadvantage (Katz et al., 2012; Sampson et al., 1997; Xue et al., 2005). Although this practice is consistent with the notion that poverty tends to be more concentrated in communities with higher proportions of African American residents, this practice precludes the examination of the diverse ways in which neighborhood racial composition may influence residents' outcomes. A particular limitation of this approach is that it negates the possibility that living in neighborhoods with higher proportions of African American residents may have salutary effects for residents. In support of this possibility, several researchers have used the percentage of African American residents in a community as a separate predictor of residents' outcomes and have found protective effects of residing in predominantly African American neighborhoods on adolescents' cigarette smoking (Reardon, Brennan, \& Buka, 2002; Xue, Zimmerman, \& Caldwell, 2007). These findings are noteworthy; however, few, if any, researchers have investigated the mechanisms through which residing in a predominantly African American neighborhood may translate into more positive youth outcomes.

One mechanism of influence linking residence in predominantly African American neighborhoods to more positive adolescent outcomes may be higher levels of social support and sense of belonging among residents in these neighborhoods. A number of studies have documented higher levels of communalism among African Americans (Boykin, Jagers, Ellison, \& Albury, 1997) and a heightened emphasis on intergenerational relationships both within and outside of the family system (Klaw, Rhodes, \& Fitzgerald, 2003; Stack, 1974). Research on the impor- tance of extended kin and fictive kin (i.e., individuals unrelated by birth or marriage but treated as family) to the African American family system suggest that child-rearing responsibilities are shared among multiple family and neighborhood adults (Stack, 1974; Stewart, 2007), thus providing increased opportunities for the formation of supportive relationships, and possibly, increasing the potential of parents to be supportive of their children through reduced caregiver burdens. Consequently, African American youth living in neighborhoods with a higher proportion of African American residents may experience greater levels of social support. Furthermore, African American youth in predominantly African American neighborhoods may perceive their neighborhoods as more supportive and feel a greater sense of connection and belonging to their neighborhood.

\section{Current Study}

This study explored pathways from neighborhood characteristics to African American adolescents' symptoms of anxiety and depression via adolescents' social support and perceptions of neighborhood cohesion. Although researchers have found protective effects of social support on adolescents' internalizing symptoms, few researchers have examined empirically whether neighborhood factors may affect adolescents' social support. Furthermore, studies of neighborhood effects on adolescents' mental health outcomes are rare, and few have included social processes and adolescents' perceptions of their neighborhoods as intervening variables. Moreover, as is common among studies of neighborhood effects on mental health outcomes, the studies conducted to date have primarily considered how neighborhood characteristics indicative of disadvantage may deleteriously affect individuals' psychological outcomes; few have included potentially cooccurring neighborhood characteristics that may predict more positive psychological outcomes. In addition, new statistical methods have emerged for studying multilevel structural or path models (Preacher, Zyphur, \& Zhang, 2010) that allow researchers to explicitly test between-group indirect effects. These methods have yet to be applied to the study of multilevel pathways from neighborhood factors to individual outcomes.

\section{Hypotheses}

This study was undertaken to address some of the limitations outlined above. Our theoretical model (see Figure 1) specifies direct and indirect effects of 


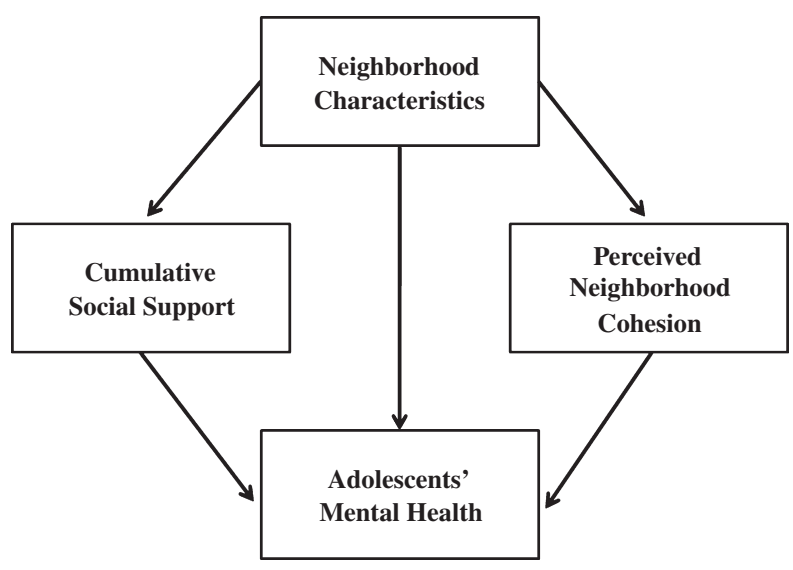

Figure 1. Hypothesized direct and indirect effects of neighborhood characteristics on adolescents' mental health.

neighborhood characteristics on African American late adolescents' symptoms of depression and anxiety. We included the following neighborhood characteristics in our study: percent of African American residents, percent of householders living in the same house for over 5 years (residential stability), percent of families living below the poverty line (poverty rate), and percent of residents over the age of 16 in the labor market but unemployed (unemployment rate). We also included a composite measure of social support, a measure of adolescents' perceptions of neighborhood cohesion, and measures of adolescents' symptoms of depression and anxiety. In addition, we included individual(gender, prior symptoms of depression and anxiety) and family-level (occupational prestige of highest earner, family structure) control variables in our model to ensure that our measurement of neighborhood factors was not just a proxy for individual or family-level factors.

We hypothesized that the percent of African American residents and residential stability of African American adolescents' neighborhoods would be positively associated with their total social support and perceptions of neighborhood cohesion. We also expected the percentage of African American residents and residential stability to negatively predict adolescents' symptoms of depression and anxiety. In contrast, we hypothesized that higher neighborhood poverty and unemployment rates would detrimentally affect adolescents' social resources and perceptions of neighborhood cohesion. We expected that higher neighborhood poverty and unemployment rates would predict more symptoms of depression and anxiety, and we hypothesized higher levels of social support and greater percep- tions of neighborhood cohesion would be associated with fewer internalizing symptoms. Thus, we expected that at least part of the hypothesized neighborhood effects on adolescents' mental health outcomes would be transmitted via social support and perceptions of neighborhood cohesion.

These hypotheses are consistent with theory and research findings regarding the detrimental effects of neighborhood poverty and unemployment on residents' social resources, perceptions of their neighborhoods, and mental health outcomes (Aneshensel \& Sucoff, 1996; Leventhal \& BrooksGunn, 2000; Massey \& Denton, 1993; Wilson, 1987), and the resource-promoting and health-protective effects of residential stability (Sampson \& Groves, 1989; Sampson \& Morenoff, 1997; Sampson et al., 1997). Notably, this is one of the first studies to explore potential mental health benefits associated with residing in neighborhoods with more African American residents and to explore pathways from neighborhood characteristics to African American adolescents' mental health via adolescents' social support and perceptions of neighborhood cohesion. Few studies have considered how potentially cooccurring neighborhood processes may differentially relate to social processes and individual outcomes. Our model acknowledges that neighborhoods are complex ecologies with the potential to exert a variety of positive and negative influences on adolescents' social and psychological outcomes.

\section{Method}

\section{Participants}

Five hundred and seventy-one African American adolescents participated in this study. Average age of participants was $17.8(S D=0.65)$ and approximately half of the sample $(52 \%)$ was female. This study is based on data collected during the fourth wave of a longitudinal study focused on high school dropout among youth in an urban, Midwestern city. The larger study recruited ninth-grade students from the four main high schools in the second largest school district in a Midwestern state. To be eligible for the study, participants needed a grade point average of 3.0 or lower at the end of the eighth grade and could not have been diagnosed by the school as having emotional or developmental impairments (the exclusion of students with impairments was done for methodological and practical purposes). The first wave of data was collected in the fall of 1994. Data were collected annually from adolescents with the first wave of data collection occurring at the 
beginning of the ninth grade. Waves 1 through 4 correspond to the participants' high school years; however, participants did not have to be enrolled in high school during Waves 2 through 4 to continue to participate in the study.

Eight hundred and fifty youth (92\% of all eligible youth) participated in Wave 1 of the larger study. Adolescents self-reporting as African American constituted $80 \%$ of the sample in Wave $1(n=681)$. We focused our analyses on data collected in Wave 4 as this was the only wave that included all of the variables of interest for our study. Our analyses only included African American participants, as we were interested in better understanding how residing in neighborhoods with more African American residents may influence positively African American adolescents' social support and perceptions of neighborhood cohesion. Of the 770 youth who participated in Wave 4 (90\% response rate from initial sample), 615 self-identified as African American. Participant data were linked to census data using geocoded home address information. Participants were excluded from our analyses if they had missing data on census-level variables (i.e., we did not have their address, we were unable to link their address to a census block group, or there were no census data associated with the block group they resided in), leaving us with our final sample of 571 African American participants. We found no differences by gender, age, socioeconomic status (SES), or Wave 1 symptoms of depression and anxiety between the 571 participants included in our analyses and the 44 participants excluded due to missing data.

\section{Procedure}

Research staff (including men and women and African American and White individuals) from local survey research organizations were hired and trained to conduct interviews with participants. The third author provided the interviewers with $20 \mathrm{hr}$ of training in administering the structured interview. Structured face-to-face interviews were conducted with students in schools or community settings if the participants could not be found in school. Interviews were always conducted in private rooms to minimize disruptions and provide maximum privacy. When possible, interviewers and participants were matched by race and gender. Interviews averaged $60 \mathrm{~min}$.

\section{Measures}

Table 1 includes ranges, means, and standard deviations for our main individual- and neighborhood-
Table 1

Participant Demographics, Ranges, Means, and Standard Deviations of Primary Study Variables at the Individual and Neighborhood Levels

\begin{tabular}{|c|c|c|c|}
\hline & Range & Mean & $\begin{array}{l}\text { Standard } \\
\text { deviation }\end{array}$ \\
\hline \multicolumn{4}{|l|}{ Participant demographics } \\
\hline Age & & 17.8 & 0.65 \\
\hline$\%$ female & & 52 & \\
\hline Family SES & & 39.45 & 9.8 \\
\hline $\begin{array}{l}\% \text { reside with } 2 \text { parental } \\
\text { figures }\end{array}$ & & 42 & \\
\hline \multicolumn{4}{|l|}{ Individual-level variables, $n=571$} \\
\hline Cumulative social support & $0-8$ & 3.71 & 2.56 \\
\hline $\begin{array}{l}\text { Perceived neighborhood } \\
\text { cohesion }\end{array}$ & $1-4$ & 2.64 & 0.99 \\
\hline $\begin{array}{l}\text { Depressive symptoms } \\
\text { (Wave 4) }\end{array}$ & $1-5$ & 1.81 & 0.91 \\
\hline Anxiety symptoms (Wave 4) & $1-5$ & 1.73 & 0.89 \\
\hline \multicolumn{4}{|c|}{ Neighborhood-level variables, $n=118$} \\
\hline$\%$ African American & $0.00-1.0$ & 0.56 & 0.37 \\
\hline$\%$ residentially stable & $0.14-0.88$ & 0.55 & 0.14 \\
\hline Poverty rate & $0.00-0.81$ & 0.26 & 0.15 \\
\hline Unemployment rate & $0.00-0.29$ & 0.10 & 0.05 \\
\hline
\end{tabular}

level variables. Of note, skewness values for all neighborhood-level variables were within \pm 1 and skewness values were within \pm 1.6 for all individuallevel variables.

\section{Neighborhood-Level Variables}

Geocoding was based on participants' residential address at the fourth wave of data collection (year: 1997) and was linked to 1990 U.S. census data (note: analyses were also conducted linking addresses to 2000 U.S. census data and findings remained consistent). Seventy-four percent of participants had been living in the same neighborhood for at least the previous three study waves. Four neighborhood-level characteristics were assessed at the block group level $(n=118$; average of 5 participants per block group): percent of African American residents, residential stability, neighborhood poverty rate, and neighborhood unemployment rate. Percent of African American residents represented the percent of the population in the block group that was African American. Residential stability was the percent of householders in the block group who had lived in their residence for over 5 years. Neighborhood poverty rate denoted the percent of families in the block group with income below the poverty level. Neighborhood unemployment rate was the percent of the labor force population in the block group who were unemployed. 


\section{Individual-Level Variables}

Social support. Our measure of social support was a cumulative measure that included support from four sources: mothers, fathers, peers, and natural mentorship. Five items were used to assess maternal support, and the same five items were reworded to assess for paternal support (adapted from Procidano \& Heller, 1983). Sample items include: I have a deep sharing relationship with my mother/father and I rely on my mother/father for emotional support. Response options ranged from 1 (not true) to 5 (very true). The five maternal support items were averaged to yield a maternal support variable $(\alpha=.91)$; the same was done for the paternal support items $(\alpha=.94)$. Five items were used to assess peer support (Procidano \& Heller, 1983). Sample items include: My friends are good at helping me solve problems and My friends give me the moral support I need. Response options ranged from 1 (not true) to 5 (very true). We computed a mean composite score to yield a peer support variable $(\alpha=.90)$. Natural mentorship was assessed with a single item: Is there an adult 25 years or older who you consider to be your mentor? That is, someone you can go to for support and guidance, or if you need to make an important decision, or who inspires you to do your best? If the participant responded in the affirmative, they were asked: What is his/her relationship to you? If participants identified a parent or stepparent as a mentor, they were asked the same question again, but asked if they could identify someone other than a parent or person who raised them.

To create our cumulative measure of social support, we first standardized the maternal support, paternal support, and peer support scales. Scores in the upper $15.9 \%$ of the distribution of each of the items ( $>1 S D$ above the mean) were designated as high levels of support, scores in the middle $68.2 \%$ were identified as average levels of support, and scores in the bottom $15.9 \%$ of the distribution (lower than $1 S D$ below the mean) were identified as low support. For each variable (maternal, paternal, and peer support), participants were assigned a score of 2 if their score on the variable was in the high range, a 1 if their score was in the average range of the distribution, and a 0 if their score was in the low range. For natural mentorship, participants were assigned a 0 if they did not identify a mentor or only identified a parent or stepparent mentor, a 1 if they identified a nonparental familial mentor, and a 2 for a nonfamilial mentor. Our decision to assign higher scores to participants with mentors outside of the family was based on the notion that having a supportive relationship with an adult outside of one's family may be indicative of a more expansive social network from which adolescents can draw additional support. Cumulative indices were computed by summing the four support scores. Accordingly, the range for the cumulative support measure was $0-8$.

Neighborhood cohesion. Five items were used to assess the extent to which participants felt their neighborhoods were supportive and cohesive (adapted from the neighborhood cohesion index; Buckner, 1988). Sample items include: The friendships I have with other people in my neighborhood mean a lot to me; If I needed advice about something, I could go to someone in my neighborhood; and I believe my neighbors would help me in an emergency. Response options ranged from 1 (strongly disagree) to 4 (strongly agree). The five items were averaged to yield one composite variable representing participants' perceptions of neighborhood cohesion $(\alpha=.74)$.

Depressive symptoms. Depressive symptoms were assessed using six items from the Brief Symptom Inventory (Derogatis \& Spencer, 1982). Participants were asked about the frequency with which they have felt uncomfortable during the past week due to problems such as feeling lonely, feeling blue (or sad), and feelings of worthlessness. Response options ranged from 1 (not at all) to 5 (extremely). The six items were averaged, with higher scores indicating higher levels of depressive symptoms $(\alpha=.87)$.

Anxiety symptoms. Six items from the Brief Symptom Inventory (Derogatis \& Spencer, 1982) were used to measure symptoms of anxiety. Participants were asked about the frequency with which they have felt uncomfortable during the past week due to problems such as nervousness or shakiness inside, feeling tense or keyed up, and feeling so restless you couldn't sit still. Response options ranged from 1 (not at all) to 5 (extremely). The six items were averaged, with higher scores indicating higher levels of anxiety symptoms $(\alpha=.89)$.

Demographic characteristics. In Wave 1, participants self-reported their race and gender. Family SES was assessed based on the highest occupational prestige score of either of the participants' parents (Nakao \& Treas, 1990). Scores for participants in this study ranged from 29.44 (private household work) to 64.38 (professional). The mean occupational prestige score was $39.45(S D=9.8)$, which represented blue-collar employment. Participants' reporting on parental occupations yielded values that were consistent with our expectations given the types of occupations available and economic 
climate of the larger community in which they resided (i.e., blue-collar, working class). To assess family structure, participants were asked who lived in their home. A dichotomous family structure variable was created: participant lived in single-parent household or with nonparental adult guardian (0) or participant lived in two-parent household with either both biological parents or one biological parent and one stepparent (1).

\section{Data Analytic Strategy}

After examining basic correlations between our variables of interest and computing intraclass correlations (ICCs) to determine whether multilevel modeling was appropriate, we proceeded to test our multilevel path model using Mplus 6 software (employing full information maximum likelihood estimation to address a limited amount of missing data; Muthén \& Muthén, 2010). Building on Muthén and Asparouhov's (2008) multilevel structural equation modeling (MSEM) mathematical framework, Preacher et al. (2010) advanced a MSEM framework for testing multilevel indirect effects. MSEM is a novel statistical approach that allows investigators to disentangle within- and between-group effects and test the significance of indirect effects occurring at the group level. Given that our analyses included nested data (individuals nested within neighborhoods) and our primary interest was in how neighborhood characteristics (Level 2 variables) predicted participants' mental health outcomes (Level 1 variables) via participants' social support and perceptions of neighborhood cohesion (both measured at Level 1), it was critical that we employed an approach that allowed us to isolate the between-group indirect effects. We did not group-mean center any of our variables because MSEM implicitly partitions each Level 1 variable into within and between components. We did not create latent factors because our main predictor variables were all based on U.S. census reports and our remaining study variables were all derived from scales with good internal reliability. Hence, our primary analysis could best be described as a multilevel path analysis testing both direct and indirect effects of neighborhood characteristics on adolescents' mental health outcomes.

Given low to moderate correlations among our neighborhood-level predictors (correlations ranged from -.04 to .49), the small number of neighborhood factors in our model, and our fairly large sample, we felt comfortable including all of these neighborhood-level variables in our model as predictors. Correlations among predictor variables in the low to moderate range are not likely to bias coefficient estimates and researchers suggest that correlations among predictor variables under .5 typically pose minimal threats to the accuracy of statistical tests (Grewal, Cote, \& Baumgartner, 2004). Our statistical model included direct and indirect paths from all of our neighborhood-level predictors to our two mental health outcome variables via adolescents' social support and perceptions of neighborhood cohesion. Our model also included correlations between all of our neighborhood-level predictor variables, and correlated error terms between social support and perceptions of neighborhood cohesion and correlated error terms between anxiety and depressive symptoms. Furthermore, we added gender, family SES, family structure, and Wave 1 symptoms of depression and anxiety as predictors of participants' social support, perceived neighborhood cohesion, and Wave 4 symptoms of depression and anxiety. We used standard goodness-of-fit indices including the chi-squared value, comparative fit index (CFI), and the root mean square error of approximation (RMSEA) to evaluate our models (Klem, 2000). We also evaluated our models based on the significance of the path coefficients with a primary focus on path coefficients corresponding to between-group effects.

\section{Results}

Correlations among study variables are shown in Table 2. The correlations were all in the directions we hypothesized; however, not all of these associations were statistically significant. Nevertheless, the correlations supported our theoretical model and demonstrated potential for testing our path model. Analysis of scatter plots did not show evidence of nonlinear associations. ICCs indicated that $7 \%$ of the variance in social support and $10 \%$ of the variance in perceptions of neighborhood cohesion could be explained by neighborhood-level differences. Additionally, 5\% of the variance in depressive symptoms and $4 \%$ of the variance in anxiety symptoms could be explained by differences across neighborhoods. These ICCs justified the use of a multilevel approach that could both account for dependency among nested observations and test group-level indirect effects. Our path model fit the data well. Although the $\chi^{2}$ test indicated a significant difference between the covariance structure implied by our model and the covariance structure of the observed data, this statis- 
Table 2

Correlations Among Study Variables

\begin{tabular}{|c|c|c|c|c|c|c|c|c|c|c|c|c|c|}
\hline & 1 & 2 & 3 & 4 & 5 & 6 & 7 & 8 & 9 & 10 & 11 & 12 & 13 \\
\hline 1. $\%$ African American & 1 & & & & & & & & & & & & \\
\hline 2. $\%$ residentially stable & $.29^{*}$ & 1 & & & & & & & & & & & \\
\hline 3. Poverty rate & $.20^{*}$ & $-.22^{*}$ & 1 & & & & & & & & & & \\
\hline 4. Unemployment rate & $.28^{*}$ & -.04 & $.49^{*}$ & 1 & & & & & & & & & \\
\hline 5. Social support & $.39 *$ & $.32^{*}$ & $-.21^{*}$ & $-.11^{*}$ & 1 & & & & & & & & \\
\hline 6. Neighborhood cohesion & $.33^{*}$ & $.40^{*}$ & $-.09^{*}$ & $-.33^{*}$ & $.23^{*}$ & 1 & & & & & & & \\
\hline 7. Gender & .01 & .02 & .01 & -.04 & .01 & $.12^{*}$ & 1 & & & & & & \\
\hline 8. Family SES & -.07 & .08 & -.04 & -.05 & .01 & -.04 & $.14^{*}$ & 1 & & & & & \\
\hline 9. Family structure & $-.09^{*}$ & .06 & -.05 & -.07 & $.10^{*}$ & .06 & $.09^{*}$ & $.12^{*}$ & 1 & & & & \\
\hline 10. Depressive symptoms w1 & -.05 & -.08 & .07 & .08 & $-.13^{*}$ & $-.16^{*}$ & $-.25^{*}$ & $-.12^{*}$ & $-.09^{*}$ & 1 & & & \\
\hline 11. Anxiety symptoms w1 & -.01 & -.05 & .05 & .03 & $-.09^{*}$ & $-.21^{*}$ & $-.20^{*}$ & $-.09^{*}$ & -.06 & $.73^{*}$ & 1 & & \\
\hline 12. Depressive symptoms w4 & $-.09^{*}$ & $-.15^{*}$ & $.09^{*}$ & .04 & $-.38^{*}$ & $-.29^{*}$ & -.08 & $.14^{*}$ & -.01 & $.25^{*}$ & $.19^{*}$ & 1 & \\
\hline 13. Anxiety symptoms w4 & -.08 & -.08 & $.10^{*}$ & .03 & $-.32^{*}$ & $-.18^{*}$ & -.08 & .06 & -.04 & $.22^{*}$ & $.21^{*}$ & $.85^{*}$ & 1 \\
\hline
\end{tabular}

Note. Gender: $0=$ female, $1=$ male; family structure: $0=$ reside with only one parental figure, one $=$ reside with two parental figures. ${ }^{*} p<.05$.

tic is easily influenced by sample size (Browne \& Cudeck, 1993). Therefore, we considered several other fit indices that are less susceptible to sample size to determine model fit. The CFI and RMSEA both indicated acceptable fit: CFI $=.97$, RMSEA $=$ $.05,95 \%$ CI $[.03, .06]$.

Figure 2 shows the unstandardized coefficients and standard errors for the statistically significant paths of interest at the between-group level. Percent African American residents in the neighborhood predicted higher levels of cumulative social support $(b=1.15, S E=0.57, p<.05)$ and greater perceptions of neighborhood cohesion $(b=0.64, S E=0.28, p<$ $.05)$ among participants. Percent of residentially stable residents in the neighborhood also predicted higher levels of social support $(b=1.22, S E=0.49$, $p<.05)$ and greater perceptions of neighborhood cohesion $(b=0.75, S E=0.34, p<.05)$ among adolescents. In contrast, neighborhood poverty rate predicted less social support $(b=-1.02, S E=0.45$, $p<.05)$, and neighborhood unemployment rate predicted reduced perceptions of neighborhood cohesion $(b=-0.48, S E=0.22, p<.05)$.

At the between-group level, adolescents' cumulative social support was negatively related to symptoms of depression $(b=-0.32, S E=0.14, p<.05)$. In addition, adolescents' perceptions of their neighborhoods as cohesive and supportive related to fewer symptoms of depression $(b=-0.20, S E=0.09$, $p<.05)$ and anxiety $(b=-0.18, S E=0.07, p<.05)$. Our analyses at the within-group level also demonstrated salubrious effects of social support and perceptions of neighborhood cohesion on depressive and anxiety symptoms; however, these within- group effects cannot be attributed to neighborhoodlevel processes and consequently cannot be used as evidence in support of our hypotheses.

Although we did not find support for direct effects of our neighborhood-level variables on our mental health outcome variables, we did find support for indirect effects via adolescents' social support and perceptions of neighborhood cohesion at the between-group level. Specifically, we found significant indirect effects of percent African American residents in the neighborhood on adolescents' depressive symptoms via adolescents' social support (unstandardized indirect effect $=-.37, S E=$ $0.16, p<.05)$ and perceptions of neighborhood cohesion (unstandardized indirect effect $=-.13, S E$ $=0.06, p<.05)$, as well as indirect effects of percent African American residents in the neighborhood on adolescents' anxiety symptoms via adolescents' perceptions of neighborhood cohesion (unstandardized indirect effect $=-.12, S E=0.05, p<.05)$. Similarly, the percent of residentially stable neighborhood residents indirectly predicted adolescents' depressive symptoms through adolescents' social support (unstandardized indirect effect $=-.39, S E=0.19$, $p<.05)$ and perceptions of neighborhood cohesion (unstandardized indirect effect $=-.15, S E=0.06$, $p<.05)$ and indirectly predicted adolescents' symptoms of anxiety via their perceptions of neighborhood cohesion (unstandardized indirect effect $=$ $-.14, S E=0.06, p<.05)$. Neighborhood poverty rate was indirectly related to adolescents' depressive symptoms via adolescents' social support (unstandardized indirect effect $=.33, S E=0.15, p<$ $.05)$ and neighborhood unemployment rate related 


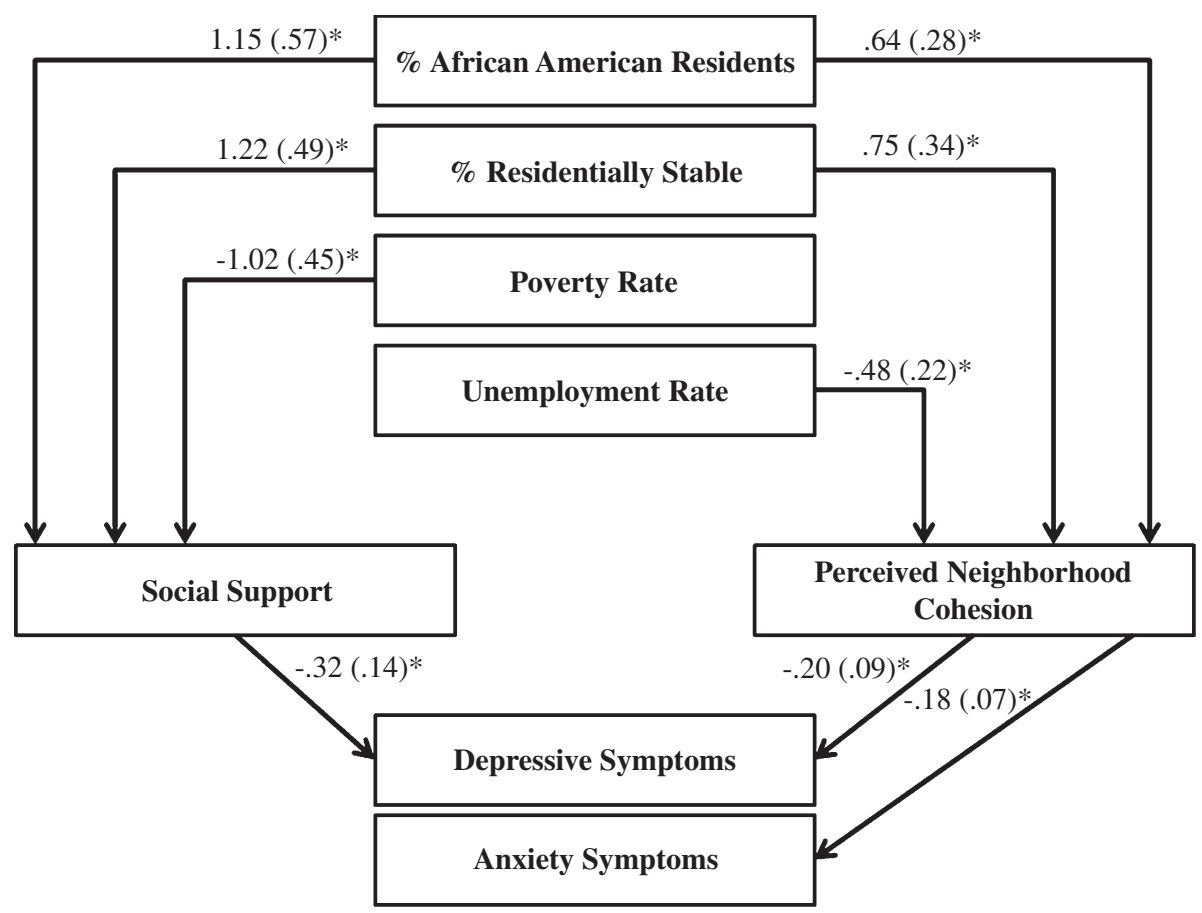

Figure 2. Results of multilevel path model analysis testing direct and indirect neighborhood effects on adolescents' symptoms of depression and anxiety adjusted for family socioeconomic status, family structure, gender, and Wave 1 symptoms of depression and anxiety.

Note. Model only displays results of between-group analysis (results of within-group analysis are available upon request). Only statistically significant paths are shown (unstandardized values) and standard errors are displayed in parentheses. Correlations between predictor variables and correlated error values among intervening variables and outcome variables are not shown but also are available upon request. $\chi^{2}(60)=158.25, p<.05, \mathrm{CFI}=.97, \mathrm{RMSEA}=.05,95 \%$ CI $[.03, .06]$.

${ }^{*} p<.05$.

to adolescents' symptoms of depression and anxiety through adolescents' perceptions of neighborhood cohesion (unstandardized indirect effect $=.10, S E=$ $0.04, p<.05$ and unstandardized indirect effect $=$ $.09, S E=0.04, p<.05$, respectively).

\section{Discussion}

Overall, study findings supported our hypotheses. Although we did not find direct effects of our neighborhood-level variables on adolescents' mental health outcomes, we did find significant indirect effects linking residence in communities with more African American and residentially stable residents with fewer symptoms of depression and anxiety among African American adolescent residents. We also found indirect effects connecting residence in communities with higher poverty and unemployment rates with more depressive and anxiety symptoms among participants. Historically, researchers have not assessed indirect pathways in the absence of direct or total effects (Baron \&
Kenny, 1986); however, a direct or total effect is not a necessary prerequisite for testing indirect effects (Hayes, 2009; Shrout \& Bolger, 2002). Neighborhood factors likely exert their influence on adolescents' mental health outcomes through multiple, complex, indirect pathways, some of which may operate in opposite directions. Informed by theory and previous research, we focused on two potential intervening variables. There are undoubtedly many other variables that could transmit the effects of neighborhood-level processes on adolescents' psychological distress. Our specific focus on social support and adolescents' perceptions of neighborhood cohesion allowed us to advance our understanding of these specific pathways; however, the absence of direct effects of our neighborhood-level variables on adolescents' symptoms of depression and anxiety suggests that there are likely other variables that we did not include in our model that also transmit the effects of our neighborhood-level variables on adolescents' psychological health (e.g., residents' perceived safety, sense of control, or levels of family conflict). 
Our findings suggesting detrimental indirect effects of neighborhood poverty and unemployment rates on adolescents' mental health are consistent with theory and previous research. This study builds on previous research by examining how these structural neighborhood attributes may contribute to adolescents' mental health problems by reducing their social resources and perceptions of neighborhood cohesion. We found that higher neighborhood poverty rates related to more depressive symptoms by potentially decreasing adolescents' cumulative social support. Also, higher neighborhood unemployment rates predicted more symptoms of anxiety and depression through reduced perceptions of neighborhood cohesion. These findings support neighborhood disadvantage theories that postulate breakdowns in social relationships as a result of neighborhood economic disadvantage (Massey \& Denton, 1993; Sampson \& Groves, 1989).

Adolescents who resided in more impoverished neighborhoods reported lower total levels of social support. Thus, experiencing higher levels of neighborhood poverty may cause residents to withdraw from their communities and consequently, receive less support from other residents. Parents may feel less able to support their own children due to their limited support in the greater community, and adolescents also may feel less able to seek out and receive support from important others in their lives (i.e., parents, peers, and nonparental adults). In light of the negative association between social support and depressive symptoms, these findings suggest that theories of social disorganization are not just important for understanding neighborhood effects on social deviance, but also may be relevant for considering neighborhood effects on mental health.

Moreover, residing in a neighborhood with a higher unemployment rate was associated with lower perceptions of neighborhood cohesion, suggesting that exposure to more unemployed adults in one's neighborhood may affect how adolescents feel about their neighborhoods. Higher neighborhood unemployment rates may be indicative of fewer working- or middle-class adult residents who are invested in their communities and actively contributing to neighborhood social capital (Wilson, 2009). With fewer adult neighbors creating and maintaining neighborhood cohesion, adolescents may feel less supported by the residents of their neighborhoods and subsequently more anxious and depressed. These findings underscore the associations between neighborhood structural attributes and residents' perceptions of their neighborhoods, as well as building on previous findings linking neigh- borhood structural characteristics to adolescents' mental health outcomes via adolescents' perceptions of their neighborhoods (Aneshensel \& Sucoff, 1996; Latkin \& Curry, 2003; Ross \& Mirowsky, 2009).

Our findings related to the health-promoting indirect effects of neighborhood residential stability on urban, African American adolescents' psychological health were in line with our hypotheses. As expected, adolescents living in neighborhoods with higher levels of residential stability reported more social support and greater perceptions of neighborhood cohesion. These findings support the notion that residentially stable neighbors are more likely to invest in social relationships in their neighborhoods (Sampson et al., 1997). This may be a case where the additional investment of some residents translates into added benefits for all neighborhood residents. As individual residents feel more supported by their larger community, they may be better able to lend support to others. Thus, higher residential stability may make social support more available and accessible to African American adolescent residents, in turn, decreasing their risk of depression.

Living in a neighborhood with a higher percentage of African American residents also yielded salubrious indirect effects on adolescents' psychological health. As we predicted, African American adolescents residing in neighborhoods with a higher percentage of African American residents reported more total social support and perceived their neighborhoods as more supportive and cohesive. These findings are consistent with previous work indicating higher levels of communalism (Boykin et al., 1997) and a greater emphasis on intergenerational relationships (Klaw et al., 2003; Stack, 1974; Stewart, 2007) within the African American community. Therefore, it is not surprising that a greater proportion of African American residents in one's neighborhood was related to reports of more total social support (and consequently, fewer symptoms of depression) and a greater sense of neighborhood cohesion (and subsequently, fewer symptoms of depression and anxiety) among African American adolescent residents. This is one of the first studies to consider the potential psychosocial benefits of residing in neighborhoods with a greater proportion of African American residents and provides an alternative perspective to scientific thought regarding the detrimental effects of living in predominantly African American, low-income, urban neighborhoods.

Wilson $(1987,2009)$ has argued that the concentration of African Americans in poor, urban neighborhoods has resulted in a lack of sustained 
interaction with mainstream society, thus preventing social and economic advancement. Furthermore, Massey and Denton (1993) suggest that the social isolation of urban African Americans may contribute to the emergence of an oppositional culture that attaches value and meaning to a way of life that mainstream society would label as deviant. According to these theories, it is not just class, but also racial segregation that exerts a negative influence on African American residents' psychosocial outcomes. Yet we found direct social and indirect mental health benefits associated with residence in neighborhoods with higher proportions of African American residents. Our findings are consistent with other research reporting positive health effects of residing in neighborhoods with greater African American racial concentration (Reardon et al., 2002; Xue et al., 2007) and research findings indicating detrimental effects of residence in areas with greater concentrations of Whites on African American adolescents' psychological health (Wickrama et al., 2005; Wight, Aneshensel, Botticello, \& Sepúlveda, 2005). Thus, continued investigations into associations between neighborhood racial composition and African American youths' psychosocial outcomes are needed to further illuminate potential patterns of influence. Findings from our study underscore the importance of considering the psychological health of neighborhood residents in addition to other indicators of adjustment.

Of note, disentangling the effects of neighborhood racial composition and neighborhood economic disadvantage has proven difficult in previous research given high correlations between the percentage of African American residents and indicators of neighborhood economic disadvantage. A benefit of this study was the relatively lower correlations between neighborhood racial composition and indicators of neighborhood economic disadvantage (i.e., poverty and unemployment rates). Given that these correlations (although positive) were relatively small, we were able to include them in our model as independent predictors of our intervening and outcome variables. This approach allowed us to identify the contrasting effects of neighborhood economic disadvantage and residence in neighborhoods with higher concentrations of African American residents on African American adolescents' psychosocial outcomes.

Notably, researchers who have investigated the effects of housing mobility and neighborhood desegregation programs (programs that aim to deconcentrate neighborhood poverty by relocating lowincome, African American and Latino families from impoverished, predominantly Black or Latino, urban neighborhoods to more affluent, predominantly White suburbs) have reported less informal contact among adults and subsequently more symptoms of depression and anxiety among African American and Latino youth who were relocated in comparison to their counterparts who remained in the same neighborhoods (Fauth, Leventhal, \& Brooks-Gunn, 2007). These findings suggest that African American families may have more informal interactions with their neighbors when residing in their predominantly Black, low-income, urban neighborhoods that are lost when they are relocated to predominantly White, middle-class suburbs. Furthermore, this loss of informal contact may contribute to more mental health problems among relocated children and adolescents. Our findings are consistent with the notion that neighborhoods with greater concentrations of African American and residentially stable residents may facilitate greater neighborhood cohesion and supportive ties, and thus promote improved psychological well-being among African American youth residents. Therefore, the findings of this study coupled with those reported by Fauth et al. (2007) suggest that policies aimed at improving the resources and economic opportunities in existing economically disadvantaged, predominantly African American neighborhoods may hold the most promise for creating more supportive communities that bolster African American adolescents' psychological health.

Collectively, the results of this study indicate that neighborhood effects may be more likely to operate through more proximal influences as opposed to exerting a direct effect on adolescents' mental health. This is consistent with the notion that neighborhood effects tend to be largely transmitted through community- and family-level processes (Leventhal \& Brooks-Gunn, 2000). Our findings suggest that additional inquiry into the ways in which neighborhoods shape adolescents' social capital and the associations between neighborhood structural attributes and adolescents' perceptions of their neighborhoods may be useful in furthering our understanding of the pathways through which adolescents are affected by their neighborhoods.

In addition, study results suggest that neighborhoods may exert positive and negative influences on adolescents' psychological outcomes. In light of the weak to moderate correlations between the neighborhood characteristics included in our study, our findings suggest that neighborhoods are complex ecologies with co-occurring attributes that differentially relate to adolescents' outcomes. The 
coexistence of neighborhood attributes that may contribute to risk and neighborhood attributes that may promote resilience belie the labeling of neighborhoods as bad or good and suggest that densely poor neighborhoods may possess a number of redeeming qualities that promote the mental wellbeing of adolescent residents. Programs and policies that are able to both reinforce health-promoting neighborhood attributes while addressing neighborhood factors that contribute to risk may be most effective in improving the quality of life of neighborhood residents.

\section{Limitations and Directions for Future Research}

Several study limitations must be noted. Although our findings of significant indirect effects of neighborhood characteristics on adolescents' symptoms of depression and anxiety indicate an association between neighborhood factors measured at the census level and youth outcomes measured at the individual level, it is important to note that our intervening and outcome variables were both measured at the individual level, introducing the possibility of shared method variance. It is possible, for example, that adolescents who are more depressed are more likely to perceive their neighborhoods negatively or withdraw from social relationships. To account for this possibility, we included previous symptoms of depression and anxiety as predictors of both intervening and outcome variables. Our findings persisted after including these variables in our model, suggesting that social support and perceptions of neighborhood cohesion were affected by structural attributes of adolescents' neighborhoods, and adolescents' experiences of social support and perceptions of neighborhood cohesion influenced their mental health. Furthermore, our statistical analysis allowed us to isolate the neighborhood-level indirect effects. Their significance lends further support to the notion that neighborhoods exerted an effect on adolescents' mental health outcomes via adolescents' social support and perceptions of neighborhood cohesion. An alternative strategy would have been to collect data on social relationships and neighborhood cohesion from other sources. Although this alternate approach addresses issues of shared method variance and is of value, taking a phenomenological approach to better understand adolescents' subjective perceptions of neighborhood cohesion and sense of support is also a worthy approach (Aneshensel \& Sucoff, 1996; Latkin \& Curry, 2003; Ross \& Mirowsky, 2009).
As is common with almost all studies of neighborhood effects, there is the possibility of selection bias (Tienda, 1991). Although we are not able to completely rule out this possibility, we controlled for family-level SES and family structure in our models as these variables may have influenced neighborhood selection. Another possible limitation is the possibility that the adults and peers adolescents were receiving support from did not reside in their neighborhoods. Older adolescents are increasingly mobile and may be more likely to form relationships outside of their neighborhoods. Nevertheless, at the least, our findings may reflect an increased ability to seek out and receive support from others both within and outside their neighborhood among youth who live in neighborhoods with higher rates of residential stability and greater concentrations of African Americans. These communities may be more supportive and communal and may teach youth the skills they need to garner support from others. This is underscored by our finding that youth in these types of neighborhoods perceived their neighborhoods as more supportive and cohesive.

In this study, we were interested in advancing our understanding of the potential of a variety of neighborhood factors to shape adolescents' cumulative experiences of social support. Thus, we standardized and compiled measures of support from various sources. Use of a measure that was designed to assess participants' cumulative support experiences would have been preferable. Nevertheless, prior to creating our cumulative variable, we found that support from mothers, fathers, peers, and the presence of a natural mentor were all correlated with our predictor and outcome variables in similar ways. Therefore, we felt that combining these measures was appropriate. Although considering differential pathways from neighborhood factors to adolescents' internalizing symptoms via support from each different source was beyond the scope of this study, future research that explores these pathways separately (as opposed to cumulatively) may highlight nuances that were not investigated in this study.

Given some of the unique attributes of participants in this study and their neighborhoods, these findings may not generalize to other groups of youth living in different types of neighborhoods. Participants in this study were African American late adolescents residing in urban neighborhoods. Approximately half of the study participants resided in neighborhoods where over 30\% of neighborhood families had incomes below the poverty level and average unemployment rate across neigh- 
borhoods was $10 \%$. Unfortunately, these neighborhood characteristics are not unlike neighborhoods in other urban centers across the United States where dense concentrations of African American youth can be found. Therefore, although not generalizable to all youth, our findings may be relevant for researchers, practitioners, and policy makers in other urban, impoverished settings. As noted above, we did not find strong correlations among our various neighborhood-level variables (e.g., percent of African American residents and neighborhood poverty rate). These lower correlations suggest that these neighborhoods may be somewhat less likely to demonstrate patterns of segregation as a function of race and class in comparison to other urban cities in the United States. While this provided a unique opportunity for us to explore differential associations between these variables and our intervening and outcome variables, these lower correlations in comparison to those found in previous neighborhood studies may be indicative of the uniqueness of the neighborhoods included in our study and further limit our ability to make generalizations from this study's findings.

This study included older adolescent participants. We expected these youth to be affected by their neighborhoods indirectly through effects on their social relationships and also through the ways they experienced their neighborhoods. Although children and younger adolescents may have fewer direct experiences with their neighborhood community, they are probably no less likely to be indirectly affected by their neighborhood through familial and peer influences. In accordance, the field can benefit from continued research on indirect pathways of neighborhood influence on youth outcomes across all developmental stages. Longitudinal studies that test evolving neighborhood effects over time will be particularly informative, especially to the extent that they examine patterns of mobility and cumulative effects of neighborhood factors on youth outcomes. Further, beyond only assessing neighborhood effects on youths' outcomes, there is a need for studies that investigate bidirectional influences between youth and their neighborhood contexts.

In spite of these limitations, our study makes a number of valuable contributions to the study of neighborhood effects on adolescent mental health. As mentioned above, this is one of the first studies to employ a multilevel path modeling approach to test neighborhood-level indirect effects on individual-level outcomes. This approach shows promise for researchers interested in testing indirect effects across multiple levels of analysis and lends itself to the study of neighborhood effects on individuals' mental health. In addition to applying this novel statistical approach, the low to moderate correlations among our census-level variables allowed us to explore the unique influence of neighborhoodlevel variables that have frequently been used as indicators of the same latent factor. Interestingly, we identified potentially opposing effects of these variables such that neighborhood poverty and unemployment rates appeared to detrimentally affect youth outcomes while residential stability and proportion of African American residents may have promoted more positive youth outcomes.

These findings highlight the need to consider the full range of potential effects of various neighborhood attributes on a variety of psychosocial outcomes, and advocate additional investigation into the role of neighborhood racial composition in facilitating healthy adolescent development. Our results suggest that there are benefits of disentangling the effects of neighborhood economic disadvantage (e.g., concentrated poverty) and racial composition, whenever possible, as these variables may relate differentially to individual outcomes. Lastly, we found evidence of new pathways through which neighborhoods may exert their influence on adolescents' mental health, after controlling for a variety of relevant individual and family variables. Our findings emphasize the merit of continued investigations into how distal neighborhood attributes may influence youth psychosocial outcomes via proximal forces, such as social relationships and subjective experiences of neighborhoods.

\section{Implications}

On the whole, our results point to the potential utility of interventions at the neighborhood level to promote improved mental health outcomes among urban, African American adolescents. Prevention efforts to date have relied heavily on interventions at the individual and family levels, with little to no attention paid to neighborhood contextual influences that may shape family and individual factors and indirectly affect mental health outcomes. Our results suggest that communitylevel interventions that address structural attributes that impede or promote social ties within the neighborhood may contribute to reduced psychological distress among urban, African American adolescents. Interventions at the community level have the potential to prevent mental health problems among larger segments of the population and have the added benefit of yielding sustainable 
results. Large-scale interventions, such as identifying or creating access to nearby employment opportunities, have the potential to reduce rates of neighborhood poverty and unemployment while simultaneously reinforcing residential stability. Results of our study indicate that these changes may foster more caring neighborhood settings that could help prevent mental health problems among adolescent residents.

Additionally, our results indicate that economically disadvantaged neighborhoods may also possess attributes that promote the well-being of youth residents. Consistent with findings that neighborhood mobility programs and policies may disrupt urban, low income, ethnic minority residents' social ties, resulting in decreased mental health among relocated adolescents (Fauth et al., 2007), we found that the African American adolescents in our study may have reaped social support benefits from living in neighborhoods with higher rates of residential stability and greater concentrations of African Americans and that these benefits may have translated into reduced psychological distress. Taken together, our findings suggest that public and private efforts to invest in urban, economically disadvantaged, predominantly African American communities by creating additional economic opportunities and promoting neighborhood stabilization may hold the most promise for improving the psychological well-being of young, urban, African American residents.

\section{References}

Aneshensel, C. S., \& Sucoff, C. A. (1996). The neighborhood context of adolescent mental health. Journal of Health and Social Behavior, 37, 293-310.

Baron, R. M., \& Kenny, D. A. (1986). The moderatormediator variable distinction in social psychological research: Conceptual, strategic, and statistical considerations. Journal of Personality and Social Psychology, 51, 1173-1182.

Bell, C. C., \& Jenkins, E. J. (1993). Community violence and children on Chicago's Southside. Psychiatry, 56, 46-54.

Boykin, A. W., Jagers, R. J., Ellison, C. M., \& Albury, A. (1997). Communalism: Conceptualization and measurement of an Afrocultural social orientation. Journal of Black Studies, 27, 409-418.

Breslau, N., Schultz, L., \& Peterson, E. (1995). Sex differences in depression: A role for preexisting anxiety. Psychiatry Research, 58, 1-12.

Bronfenbrenner, U. (1979). The ecology of human development: Experiments by nature and design. Cambridge, MA: Harvard University Press.
Brooks-Gunn, J., Duncan, G. J., Leventhal, T., \& Aber, J. L. (1997). Lessons learned and future directions for research on the neighborhoods in which children live. In J. Brooks-Gunn, G. J. Duncan, \& J. L. Aber (Eds.), Neighborhood poverty: Contexts and consequences for children (Vol. 1, pp. 279-297). New York: Russell Sage Foundation.

Browne, M. W., \& Cudeck, R. (1993). Alternative ways of assessing model fit. In K. A. Bollen \& J. S. Long (Eds.), Testing structural equation models (pp. 136-162). Newbury Park, CA: Sage.

Buckner, J. (1988). The development of an instrument to measure neighborhood cohesion. American Journal of Community Psychology, 16, 771-791.

Colarossi, L. G., \& Eccles, J. S. (2003). Differential effects of support providers on adolescents' mental health. Social Work Research, 27, 19-30.

Coleman, J. S. (1988). Social capital in the creation of human capital. American Journal of Sociology, 94, S95-S121.

Cook, T. D., Shagle, S. C., \& Degirmencioglu, S. M. (1997). Capturing social process for testing meditational models of neighborhood effects. In J. Brooks-Gunn, G. J. Duncan, \& J. L. Aber (Eds.), Neighborhood poverty: Policy implications in studying neighborhoods (Vol. 2, pp. 94-119). New York: Russell Sage Foundation.

Deng, S., Lopez, V., Roosa, M. W., Ryu, E., Burrell, G. L., Tein, J.-Y., et al. (2006). Family processes mediating the relationship of neighborhood disadvantage to early adolescent internalizing problems. Journal of Early Adolescence, 26, 206-231.

Derogatis, L. R., \& Spencer, P. M. (1982). The Brief Symptom Inventory (BSI): Administration and scoring procedures. Baltimore: Division of Medical Psychology, John Hopkins University School of Medicine.

Dominguez, S., \& Watkins, C. (2003). Creating networks for survival and mobility: Social capital among African American and Latin-American low-income mothers. Social Problems, 50, 111-135.

Duncan, G. J., Brooks-Gunn, J., \& Klebanov, P. K. (1994). Economic deprivation and early childhood development. Child Development, 65, 296-318.

Fauth, R. C., Leventhal, T., \& Brooks-Gunn, J. (2007). Welcome to the neighborhood? Long-term impacts of moving to low-poverty neighborhoods on poor children's and adolescents' outcomes. Journal of Research on Adolescence, 17, 249-284.

Furstenberg, F. F. Jr. (1993). How families manage risk and opportunity in dangerous neighborhoods. In W. J. Wilson (Ed.), Sociology and the public agenda (pp. 231258). Newbury Park, CA: Sage.

Garnefski, N., \& Diekstra, R. (1996). Perceived social support from family, school, and peers: Relationship with emotional and behavioral problems among adolescents. Journal of the American Academy of Child and Adolescent Psychiatry, 35, 1657-1664.

Geis, K. J., \& Ross, C. E. (1998). A new look at urban alienation: The effect of neighborhood disorder on perceived powerlessness. Social Psychology Quarterly, 61, 232-246. 
Gore, S., \& Aseltine, R. H. (2003). Race and ethnic differences in depressed mood following the transition from high school. Journal of Health and Social Behavior, 44, 370-389.

Gotlib, I. H., Lewinsohn, P. M., \& Seeley, J. R. (1995). Symptoms versus a diagnosis of depression: Differences in psychosocial functioning. Journal of Consulting and Clinical Psychology, 63, 90-100.

Grewal, R., Cote, J. A., \& Baumgartner, H. (2004). Multicollinearity and measurement error in structural equation models: Implications for theory testing. Marketing Science, 23, 519-529.

Hayes, A. F. (2009). Beyond Baron and Kenny: Statistical mediation analysis in the new millennium. Communication Monographs, 76, 408-420.

Himle, J. A., Baser, R. E., Taylor, R. J., Campbell, R. D., \& Jackson, J. S. (2009). Anxiety disorders among African Americans, blacks of Caribbean descent, and nonHispanic Whites in the United States. Journal of Anxiety Disorders, 23, 578-590.

Hussong, A. (2000). Perceived peer context and adolescent adjustment. Journal of Research on Adolescence, 10, 391-415.

Jargowsky, P. A. (2005). Stunning progress, hidden problems: The dramatic decline of concentrated poverty in the 1990s. In A. Berube, B. Katz, \& R. E. Lang (Eds.), Redefining urban and suburban America: Evidence from census 2000 (Vol. 2, pp. 137-171). Washington, DC: Brookings Institution Center on Urban and Metropolitan Policy.

Katz, B. N., Esparza, P., Carter, J. S., Grant, K. E., \& Meyerson, D. A. (2012). Intervening processes in the relationship between neighborhood characteristics and psychological symptoms in urban youth. Journal of Early Adolescence, 32, 650-680. doi:10.1177/0272431 611414060

Klaw, E. L., Rhodes, J. E., \& Fitzgerald, L. F. (2003). Natural mentors in the lives of African American adolescent mothers: Tracking relationships over time. Journal of Youth and Adolescence, 32, 223-232.

Klem, L. (2000). Structural equation modeling. In L. G. Grimm \& P. R. Yarnold (Eds.), Reading and understanding multivariate statistics (pp. 227-257). Washington, DC: American Psychological Association.

Kohen, D. E., Leventhal, T., Dahinten, V. S., \& McIntosh, C. N. (2008). Neighborhood disadvantage: Pathways of effects for young children. Child Development, 79, 156169.

Latkin, C. A., \& Curry, A. D. (2003). Stressful neighborhoods and depression: A prospective study of the impact of neighborhood disorder. Journal of Health and Social Behavior, 44, 34-44.

Leventhal, T., \& Brooks-Gunn, J. (2000). The neighborhoods they live in: The effects of neighborhood residence on child and adolescent outcomes. Psychological Bulletin, 126, 309-337.

Lewinsohn, P. M., Hops, H., Roberts, R. E., Seeley, J. R., \& Andrews, J. A. (1993). Adolescent psychopathology:
I. Prevalence and incidence of depression and other DSM-III-R disorders in high school students. Journal of Abnormal Psychology, 102, 133-134.

Massey, D. S., \& Denton, N. A. (1993). American apartheid: Segregation and the making of the underclass. Cambridge, MA: Harvard University Press.

Munsch, J., \& Blyth, D. A. (1993). An analysis of the functional nature of adolescents' supportive relationships. Journal of Early Adolescence, 13, 132-153.

Murry, V. M., Berkel, C., Gaylord-Harden, N. K., Copeland-Linder, N., \& Nation, M. (2011). Neighborhood poverty and adolescent development. Journal of Research on Adolescence, 21, 114-128.

Muthén, B. O., \& Asparouhov, T. (2008). Growth mixture modeling: Analysis with non-Gaussian random effects. In G. Fitzmaurice, M. Davidian, G. Verbeke, \& G. Molenberghs (Eds.), Longitudinal data analysis (pp. 143-165). Boca Raton, FL: Chapman \& Hall/CRC.

Muthén, L. K., \& Muthén, B. O. (2010). Mplus user's guide (6th ed.) [Computer software and manual]. Los Angeles: Muthén \& Muthén.

Nakao, K., \& Treas, J. (1990). The 1989 socioeconomic index of occupations: Construction of the 1989 occupational prestige scores. (GSS Methodological Report No. 74). Chicago: National Opinion Research Center.

Newman, B. M., Newman, P. R., Griffen, S., O'Connor, K., \& Spas, J. (2007). The relationship of social support to depressive symptoms during the transition to high school. Adolescence, 42, 441-459.

Pierce, R. S., Frone, M. R., Russell, M., Cooper, M. L., \& Mudar, P. (2000). A longitudinal model of social contact, social support, depression and alcohol use. Health Psychology, 19, 28-38.

Pinderhughes, E. E., Nix, R., Foster, E. M., \& Jones, D. (2001). Parenting in context: Impact of neighborhood poverty, residential stability, public services, social networks, and danger on parental behaviors. Journal of Marriage and Family, 63, 941-953.

Preacher, K. J., Zyphur, M. J., \& Zhang, Z. (2010). A general multilevel SEM framework for assessing multilevel mediation. Psychological Methods, 15, 209-233.

Procidano, M. E., \& Heller, K. (1983). Measures of perceived social support from friends and from family: Three validation studies. American Journal of Community Psychology, 11, 1-24.

Rao, U., Hammen, C., \& Daley, S. E. (1999). Continuity of depression during the transition to adulthood: A 5-year longitudinal study of young women. Journal of the American Academy of Child and Adolescent Psychiatry, 38, 908-915.

Reardon, S. F., Brennan, R. T., \& Buka, S. L. (2002). Estimating multi-level discrete-time hazard models using cross-sectional data: Neighborhood effects on the onset of adolescent cigarette use. Multivariate Behavioral Research, 37, 297-330.

Ross, C. E., \& Mirowsky, J. (2009). Neighborhood disorder, subjective alienation, and distress. Journal of Health and Social Behavior, 50, 49-64. 
Sampson, R. J., \& Groves, W. B. (1989). Community structure and crime: Testing social disorganization theory. American Journal of Sociology, 94, 774-802.

Sampson, R. J., \& Morenoff, J. D. (1997). Ecological perspectives on the neighborhood context of urban poverty: Past and present. In J. Brooks-Gunn, G. J. Duncan, \& J. L. Aber (Eds.), Neighborhood poverty: Policy implications in studying neighborhoods (Vol. 2, pp. 1-22). New York: Russell Sage Foundation.

Sampson, R. J., Raudenbush, S. W., \& Earls, F. (1997). Neighborhoods and violent crime: A multilevel study of collective efficacy. Science, 277, 918-924.

Shrout, P. E., \& Bolger, N. (2002). Mediation in experimental and nonexperimental studies: New procedures and recommendations. Psychological Methods, 7, 422-445.

Small, M. L. (2007). Racial differences in networks: Do neighborhood conditions matter? Social Science Quarterly, 88, 320-343.

Stack, C. B. (1974). All our kin: Strategies for survival in a Black community. New York: Harper \& Row.

Stewart, P. (2007). Who is kin? Family definition and African American families. Journal of Human Behavior in the Social Environment, 15, 163-181.

Tienda, M. (1991). Poor people, poor places: Deciphering neighborhood effects on poverty outcomes. In J. Huber (Ed.), Macro-micro linkages in sociology (pp. 244-262). Newbury Park, CA: Sage.

Turner, R. J., \& Brown, R. L. (2010). Social support and mental health. In T. L. Sheid \& T. N. Brown (Eds.), A handbook for the study of mental health: Social contexts, theories, and systems (2nd ed., pp. 200-212). Cambridge, UK: Cambridge University Press.

Upchurch, D. M., Aneshensel, C. S., Sucoff, C. A., \& Levy-Storms, L. (1999). Neighborhood and family contexts of adolescent sexual activity. Journal of Marriage and Family, 61, 920-933.

Wickrama, K. A. S., \& Bryant, C. (2003). Community context of social resources and adolescent mental health. Journal of Marriage and Family, 65, 850-866.
Wickrama, K. A. S., Noh, S., \& Bryant, C. M. (2005). Racial differences in adolescent distress: Differential effects of the family and community for Blacks and Whites. Journal of Community Psychology, 33, 261-282.

Wight, R. G., Aneshensel, C. S., Botticello, A. L., \& Sepúlveda, J. E. (2005). A multilevel analysis of ethnic variation in depressive symptoms among adolescents in the United States. Social Science E Medicine, 60, 2073-2084.

Williams, D. R., Gonzalez, H. M., Neighbors, H., Nesse, R., Abelson, J. M., Sweetman, J., et al. (2007). Prevalence and distribution of major depressive disorder in African Americans, Caribbean blacks, and non-Hispanic Whites: Results from the National Survey of American Life. Archives of General Psychiatry, 64, 305-315.

Wilson, W. J. (1987). The truly disadvantaged: The inner city, the underclass, and public policy. Chicago: University of Chicago Press.

Wilson, W. J. (2009). More than just race: Being Black and poor in the inner city. New York: Norton.

Xue, Y., Leventhal, T., Brooks-Gunn, J., \& Earls, F. J. (2005). Neighborhood residence and mental health problems of 5- to 11-year-olds. Archives of General Psychiatry, 62, 554-563.

Xue, Y., Zimmerman, M. A., \& Caldwell, C. H. (2007). Neighborhood residence and cigarette smoking among urban youths: The protective role of prosocial activities. American Journal of Public Health, 97, 1865-1872.

Zahn-Waxler, C., Klimes-Dougan, B., \& Slattery, M. J. (2000). Internalizing problems of childhood and adolescence: Prospects, pitfalls, and progress in understanding the development of anxiety and depression. Development and Psychopathology, 12, 443-466.

Zimmerman, M. A., Ramírez-Valles, J., \& Maton, K. I. (1999). Resilience among urban African American male adolescents: A study of the protective effects of sociopolitical control on their mental health. American Journal of Community Psychology, 27, 733-751. 